CASE REPORT

\title{
Fetal Intra-abdominal Umbilical Vein Varix Assessed by the Novel Doppler Ultrasound
}

Toshiyuki Hata ${ }^{1}$, Aya Koyanagi ${ }^{2}$, Tomomi Yamanishi ${ }^{3}$, Saori Bouno ${ }^{4}$, Riko Takayoshi ${ }^{5}$, Takahiro Ohkura ${ }^{6}$, Yasuo Nakahara ${ }^{7}$, Takahito Miyake ${ }^{8}$

\begin{abstract}
We present two cases of fetal intra-abdominal umbilical vein varix (FIUVV) assessed using RadiantFlow, HDlive Flow, and SlowflowHD. In the first case, FIUVV may have been caused by a persistent right umbilical vein, and it may have been due to umbilical ring contracture in the second case. These causes were suspected by the novel Doppler ultrasound. These novel Doppler techniques may provide useful information on the prenatal diagnosis and understanding of the pathophysiology of FIUVV.
\end{abstract}

Keywords: Fetal intra-abdominal umbilical vein varix, HDlive Flow, Pathophysiology, Prenatal diagnosis, RadiantFlow, SlowflowHD.

Donald School Journal of Ultrasound in Obstetrics and Gynecology (2020): 10.5005/jp-journals-10009-1672

\section{INTRODUCTION}

The incidence of fetal intra-abdominal umbilical vein varix (FIUVV) is 0.1 to 2.8 per 1,000 pregnancies. ${ }^{1,2}$ Isolated FIUVV can be easily diagnosed using color/power Doppler ultrasound and is associated with a favorable prognosis. ${ }^{3,4}$ There have been several studies on three-dimensional (3D) power Doppler ultrasound assessment of FIUVV during pregnancy. ${ }^{5-7}$ Using this technique, we can clearly recognize the spatial relationships between FIUVV and surrounding vasculatures. HDlive Flow is one form of 3D color/power Doppler that facilitates the spatial reconstruction of the fetal heart and blood vessels with an adjustable light source to realize lighting and shadowing effects. ${ }^{8-14}$

RadiantFlow is a unique form of Doppler ultrasound, which shows 3D color/power Doppler information on a two-dimensional (2D) grayscale image by shading based on the amplitude of the color/power Doppler signal. ${ }^{15-17}$

SlowflowHD is a novel power Doppler technique that can depict slow blood flow in fetal peripheral circulations. ${ }^{18,19}$ In this study, we present two cases of FIUVV assessed using RadiantFlow, HDlive Flow, and SlowflowHD.

\section{Case Description}

\section{Case 1}

A 40-year-old pregnant Japanese woman, gravida 1, para 0, received a routine third-trimester fetal anomaly scan at 29 weeks and 3 days of gestation, and round FIUVV (13.6 mm) was noted by color Doppler ultrasound (Fig. 1). Swirling flow in the varix was identified using a power Doppler ultrasound (Fig. 2). SlowflowHD showed a persistent right umbilical vein making a right-angled turn from FIUVV (Fig. 3). HDlive Flow clearly demonstrated spatial relationships among vortex FIUVV flow and surrounding blood vessels (Fig. 4). The size of FIUVV did not change during pregnancy, and we could not find the clot in FIUVV.

At 38 weeks and 1 day of gestation, the elective cesarean section was performed due to previous uterine surgery, resulting in a viable, single male newborn weighing $2,804 \mathrm{~g}$, with a height of

\footnotetext{
1,8Department of Obstetrics and Gynecology, Miyake Clinic, Ofuku, Minami-ku, Okayama, Japan; Department of Perinatology and Gynecology, Kagawa University Graduate School of Medicine, Ikenobe, Miki, Kagawa, Japan

${ }^{2-5}$ Department of Obstetrics and Gynecology, Miyake Clinic, Ofuku, Minami-ku, Okayama, Japan

${ }^{6,7}$ Department of Pediatric Surgery, Okayama Medical Center, Tamasu, Kita-ku, Okayama, Japan
}

Corresponding Author: Toshiyuki Hata, Department of Obstetrics and Gynecology, Miyake Clinic, Ofuku, Minami-ku, Okayama, Japan; Department of Perinatology and Gynecology, Kagawa University Graduate School of Medicine, Ikenobe, Miki, Kagawa, Japan, Phone: +81-(0)87-891-2174, e-mail: toshi28@med.kagawa-u.ac.jp

How to cite this article: Hata T, Koyanagi A, Yamanishi T, et al. Fetal Intra-abdominal Umbilical Vein Varix Assessed by the Novel Doppler Ultrasound. Donald School J Ultrasound Obstet Gynecol 2020;14(4): 342-345.

Source of support: Nil

Conflict of interest: None

$48 \mathrm{~cm}$. The Apgar scores were 9 (1 minute) and 10 (5 minutes), and the umbilical artery blood $\mathrm{pH}$ was 7.39 . The mother and neonate followed a favorable course after the delivery.

\section{Case 2}

A 36-year-old pregnant Japanese woman, gravida 1, para 0, received a routine third-trimester fetal anomaly scan at 30 weeks of gestation, and tubular FIUVV was noted by 2D sonography (Fig. 5). RadiantFlow showed two-stream flows in the tubular FIUVV (Fig. 6), and a high-peak velocity was noted (Fig. 7). SlowflowHD depicted precise intrahepatic small vessels (Fig. 8). HDlive Flow clearly demonstrated umbilical ring contracture (Fig. 9). The size of FIUVV did not change during pregnancy, and we could not find the clot in FIUVV.

One male newborn was vaginally delivered at 40 weeks and 5 days of gestation with a body weight of $2,952 \mathrm{~g}$ and a

() The Author(s). 2020 Open Access This article is distributed under the terms of the Creative Commons Attribution 4.0 International License (https://creativecommons. org/licenses/by-nc/4.0/), which permits unrestricted use, distribution, and non-commercial reproduction in any medium, provided you give appropriate credit to the original author(s) and the source, provide a link to the Creative Commons license, and indicate if changes were made. The Creative Commons Public Domain Dedication waiver (http://creativecommons.org/publicdomain/zero/1.0/) applies to the data made available in this article, unless otherwise stated. 


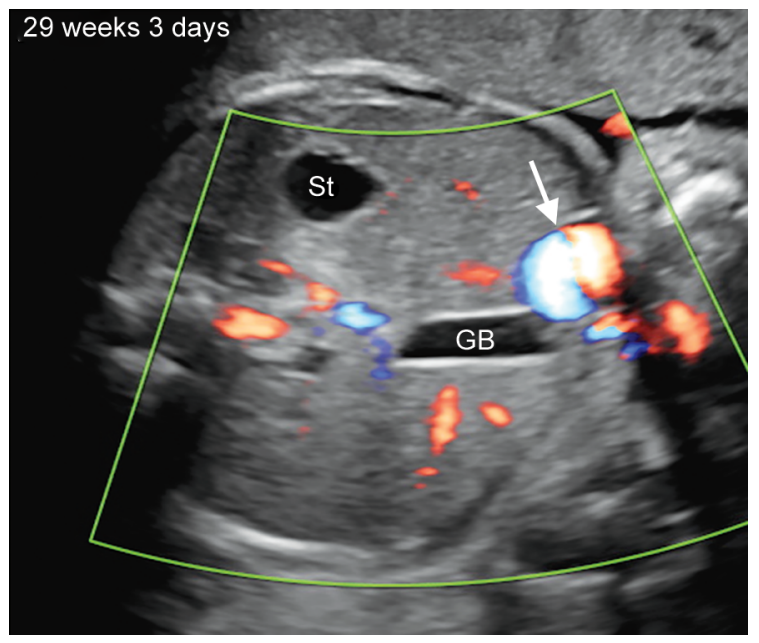

Fig. 1: Color Doppler ultrasound image of fetal intra-abdominal umbilical vein varix (arrow) at 29 weeks and 3 days of gestation. GB, gallbladder; St, stomach

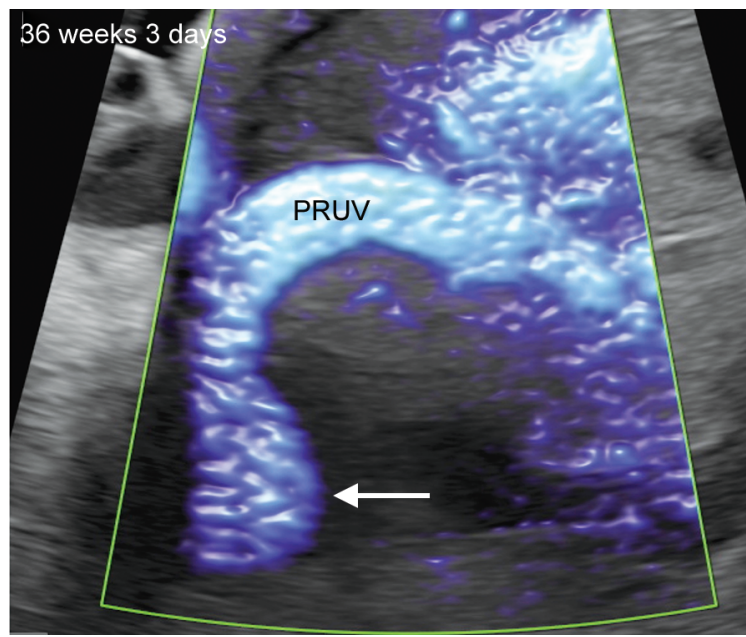

Fig. 3: SlowflowHD image of fetal intra-abdominal umbilical vein varix (arrow) at 36 weeks and 3 days of gestation. PRUV, persistent right umbilical vein

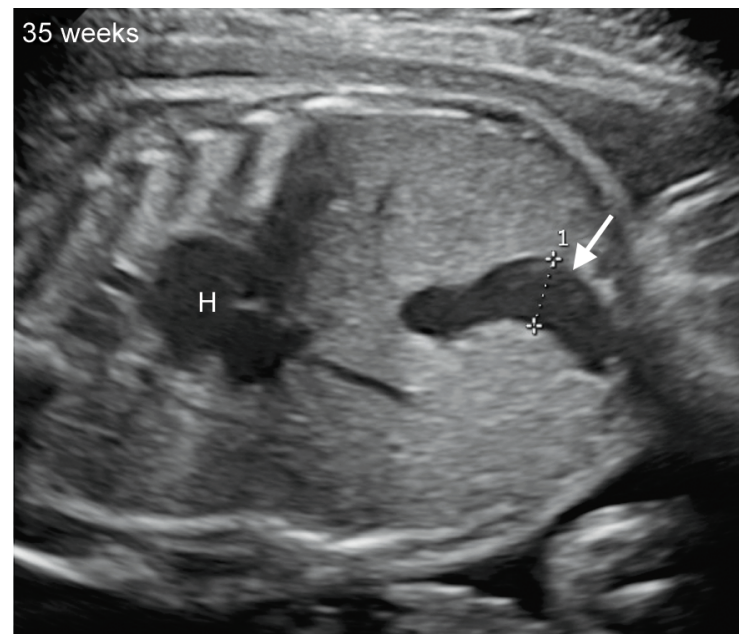

Fig. 5: Two-dimensional sonographic image of fetal intra-abdominal umbilical vein varix (arrow) at 30 weeks of gestation. $\mathrm{H}$, heart

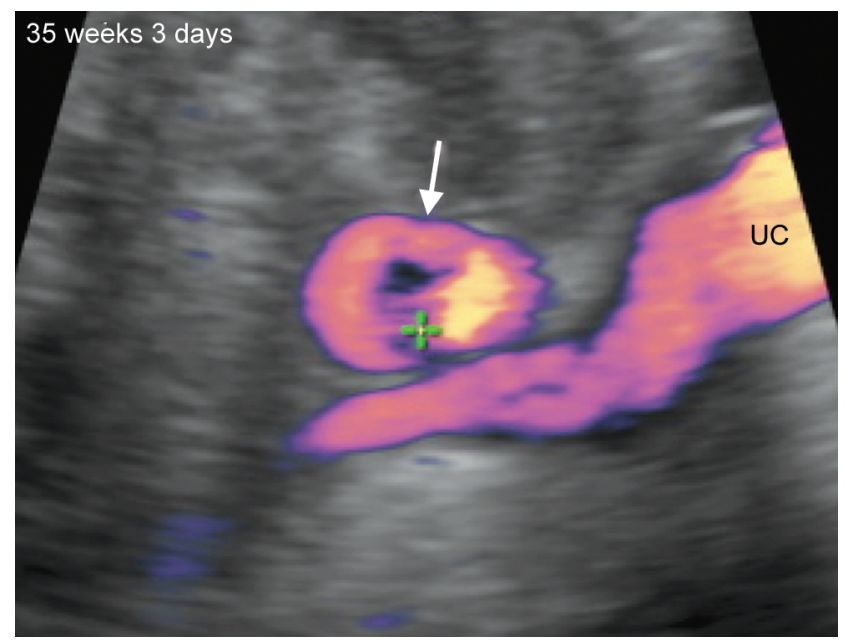

Fig. 2: Power Doppler ultrasound image of fetal intra-abdominal umbilical vein varix (arrow) at 35 weeks and 3 days of gestation. Swirling flow in the varix is noted. UC, umbilical cord

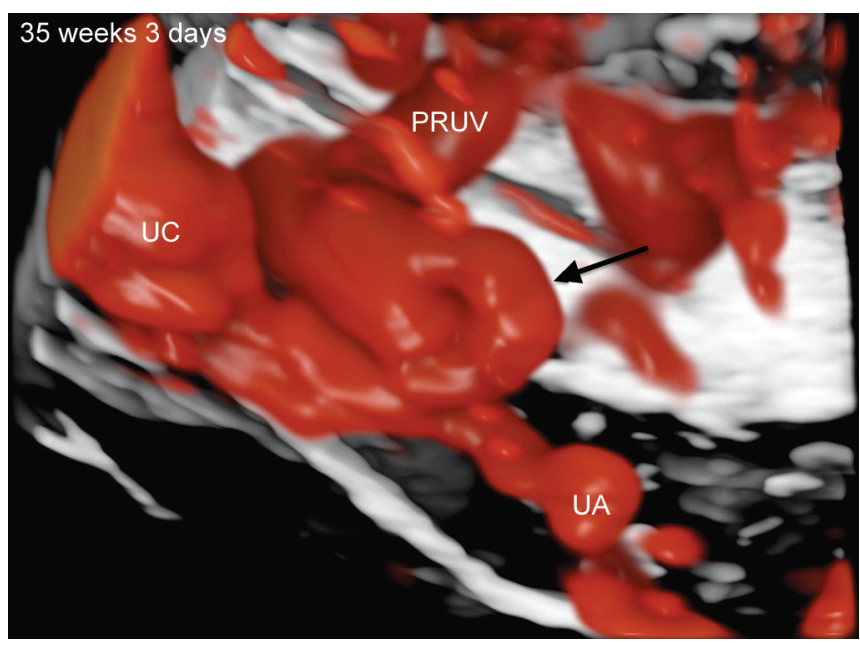

Fig. 4: HDlive Flow image of fetal intra-abdominal umbilical vein varix (arrow) at 35 weeks and 3 days of gestation. Swirling flow in the varix is spatially evident. PRUV, persistent right umbilical vein; UA, umbilical artery; UC, umbilical cord

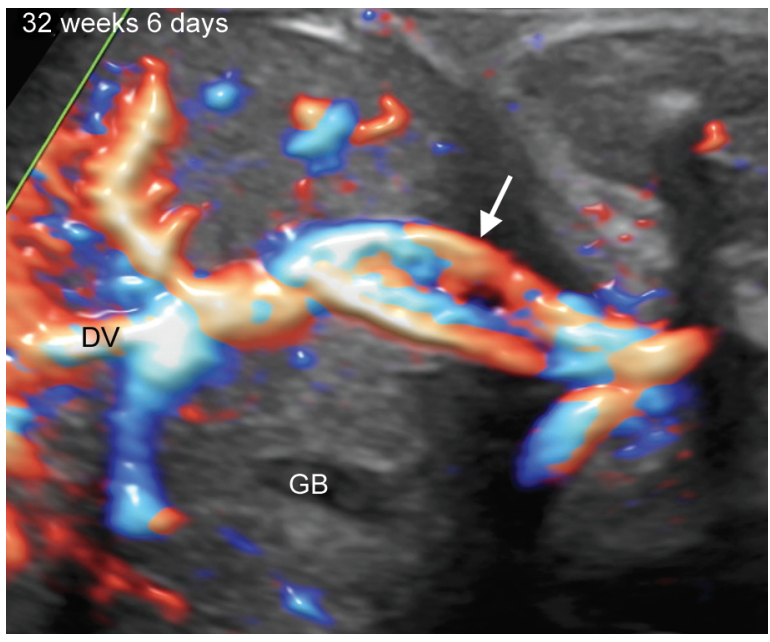

Fig. 6: RadiantFlow image of fetal intra-abdominal umbilical vein varix (arrow) at 32 weeks and 6 days of gestation. DV, ductus venosus; GB, gallbladder 


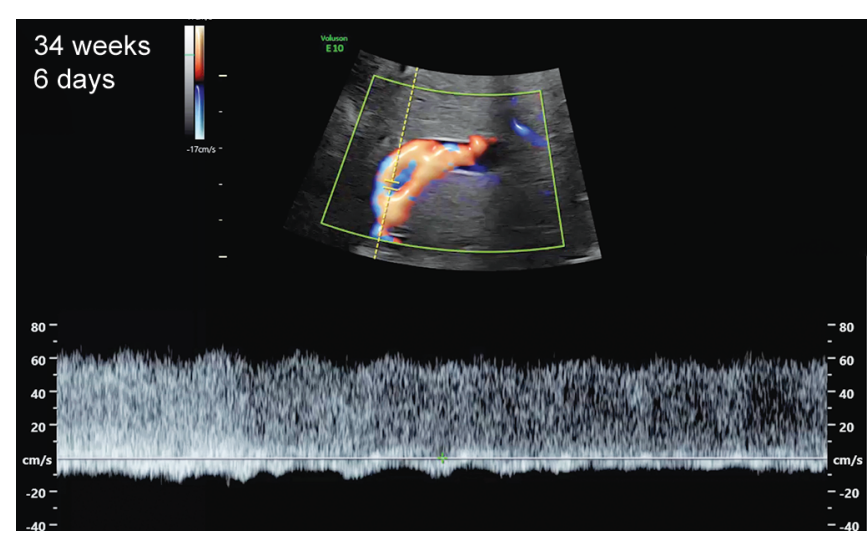

Fig. 7: Pulsed Doppler ultrasound image of fetal intra-abdominal umbilical vein varix at 34 weeks and 6 days of gestation. High-peak velocity $(60 \mathrm{~cm} / \mathrm{s})$ is evident

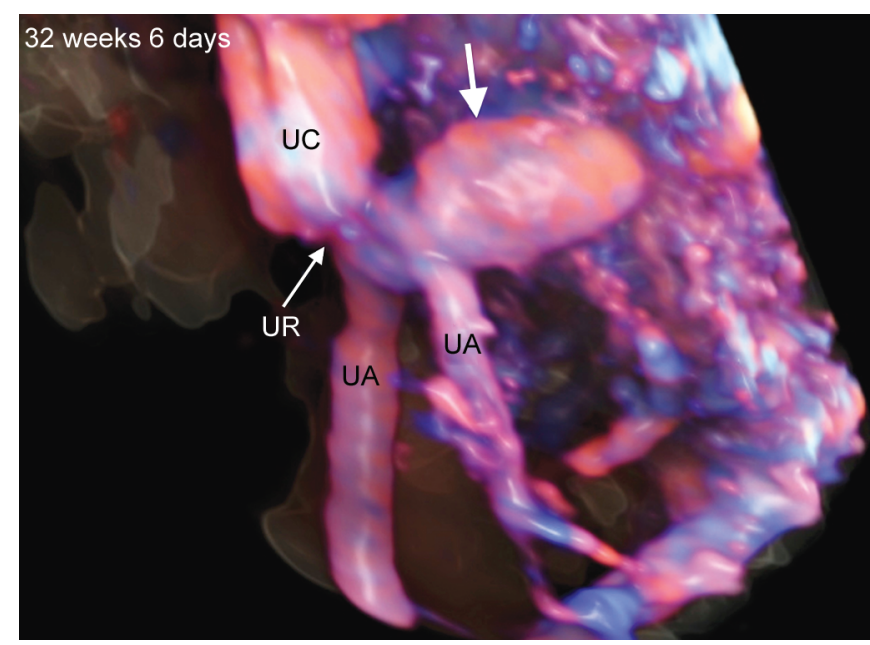

Fig. 9: HDlive Flow image of fetal intra-abdominal umbilical vein varix (arrow) at 32 weeks and 6 days of gestation. Umbilical ring contracture (UR) is clearly identified. UA, umbilical artery; UC, umbilical cord

length of $50 \mathrm{~cm}$. The umbilical artery $\mathrm{pH}$ was 7.27. He had an Apgar score of $9 / 10$ at 1 and 5 minutes, respectively. The mother and neonate followed a favorable course after the delivery.

\section{Discussion}

There have been numerous studies on the clinical characteristics of FIUVV. ${ }^{1-4,20,21}$ However, the etiology and pathophysiologic causes of FIUVV were not described in these previous studies. In our first case, straight umbilical venous flow through the umbilical ring may hit the intra-abdominal venous wall, which bends at a right angle due to the persistent right umbilical vein. Moreover, swirling flow in varix may widen FIUVV. Therefore, FIUVV may adopt a round shape in this case. In the second case, high-speed umbilical venous flow through the contracted umbilical ring may widen the intraabdominal umbilical vein. So, FIUVV may adopt a tubular shape in the second case. These pathophysiologic causes of FIUVV were suspected by novel Doppler ultrasound involving RadiantFlow, HDlive Flow, and SlowflowHD. This allows us to clearly recognize spatial relationships among FIUVV and surrounding blood vessels. These novel Doppler techniques may provide useful information on

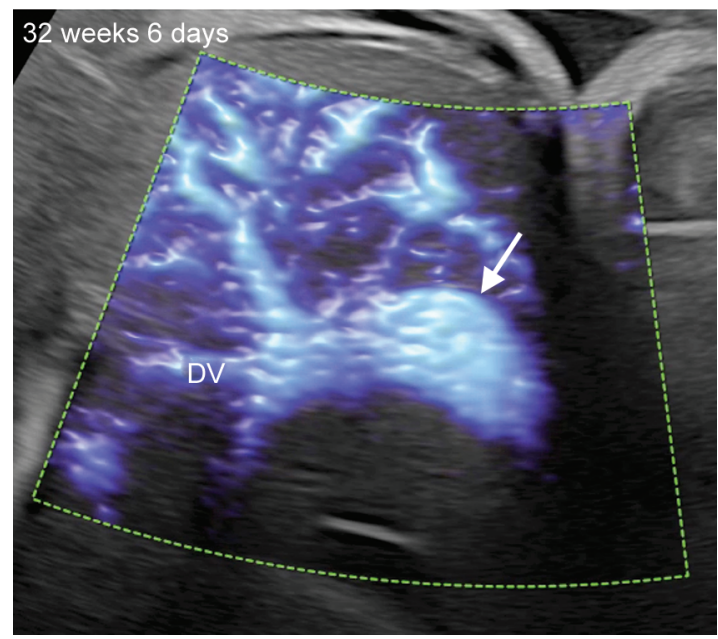

Fig. 8: SlowflowHD image of fetal intra-abdominal umbilical vein varix (arrow) at 32 weeks and 6 days of gestation. DV, ductus venosus

the prenatal diagnosis and understanding of the pathophysiology of FIUVV.

With respect to the perinatal management of FIUVV, fetal ultrasound follow-up is mandatory, focusing on an increase in the size of varix and appearance of a clot. ${ }^{2}$ In our cases, the sizes of varix did not change until delivery. Moreover, we could not detect any clots in varix. Consequently, the newborn babies followed a favorable after delivery. Novel Doppler ultrasound might also be useful for monitoring fetal well-being, especially for detecting a clot in varix.

\section{References}

1. Lee SW, Kim MY, Kim JE, et al. Clinical characteristics and outcomes of antenatal fetal intra-abdominal umbilical vein varix detection. Obstet Gynecol Sci 2014;57(3):181-186. DOI: 10.5468/ogs.2014.57.3.181.

2. Beraud E, Rozel C, Milon J, et al. Umbilical vein varix: Importance of antenatal post-natal monitoring by ultrasound. Diagn Interv Imag 2015;96(1):21-26. DOI: 10.1016/j.diii.2014.01.009.

3. Di Pasquo E, Kuleva M, O'gorman N, et al. Fetal intra-abdominal umbilical vein varix: retrospective cohort study and systematic review and meta-analysis. Ultrasound Obstet Gynecol 2018;51:580-585.

4. Novoa V, Shazly S, Ibirogba ER, et al. Perinatal outcomes of fetal intraabdominal umbilical vein varix: a multicenter cohort study. J Matern Fetal Neonatal Med 2019;17:1-4. DOI: 10.1080/14767058.2019.1685969.

5. Sciaky-Tamir Y, Cohen SM, Hochner-Celnikier D, et al. Threedimensional power Doppler (3DPD) ultrasound in the diagnosis and follow-up of fetal vascular anomalies. Am J Obstet Gynecol 2006;194(1):274-281. DOI: 10.1016/j.ajog.2005.08.045.

6. Lu M, Kakani N, Romagnoli C, et al. Two- and three-dimensional sonographic diagnosis of fetal intra-abdominal umbilical vein varix: a case report. J Clin Ultrasound 2012;40(9):586-589. DOI: 10.1002/ jcu. 21880.

7. Austria BS, Hanaoka U, Sato M, et al. Three- and four-dimensional sonographic diagnosis of fetal intra-abdominal umbilical vein varix. J Med Ultrasonics 2014;41(2):245-246. DOI: 10.1007/s10396-0130481-0.

8. AboEllail MAM, Kanenishi K, Tenkumo C, et al. Diagnosis of trncus arteriosus in first trimester of pregnancy using transvaginal fourdimensional color Doppler ultrasound. Ultrasound Obstet Gynecol 2015;45(6):759-760. DOI: 10.1002/uog.14868.

9. AboEllail MAM, Kanenishi K, Tenkumo $C$, et al. Four-dimensional power Doppler sonography with the HDlive silhouette mode in 
antenatal diagnosis of a right aortic arch with an aberrant left subclavian artery. J Ultrasound Med 2016;35(3):661-663. DOI: 10.7863/ ultra.15.05047.

10. Yang PY, Sajapala S, Yamamoto K, et al. Antenatal diagnosis of idiopathic dilatation of fetal pulmonary artery with 3D power Doppler imaging. J Clin Ultrasound 2017;45(2):121-123. DOI: 10.1002/ jcu.22367.

11. Ito $M, A$ AboEllail MAM, Yamamoto $K$, et al. HDlive flow silhouette mode and spatiotemporal image correlation for diagnosing congenital heart disease. Ultrasound Obstet Gynecol 2017;50(3):411-415. DOI: 10.1002/uog.17519.

12. Hata $T$, Ito M, Nitta E, et al. HDlive flow silhouette mode for diagnosis of ectopia cordis with a left ventricular diverticulum at 15 weeks' gestation. J Ultrasound Med 2018;37(10):2465-2467. DOI: 10.1002/ jum.14583.

13. Tenkumo C, Hanaoka U, AboEllail MAM, et al. HDlive flow with HDlive silhouette mode in diagnosis of fetal hepatic hemangioma. Ultrasound Obstet Gynecol 2017;49(4):540-545. DOI: 10.1002/ uog. 16215.

14. Hata T, Koyanagi A, Yamanishi T, et al. Success rate of five cardiac views using HDlive flow with spatiotemporal image correlation at 18-21 and 28-31 weeks of gestation. J Perinat Med 2020;48(4):384-388. DOI: 10.1515/jpm-2019-0434.
15. Hata T, Kanenishi K, Nitta E, et al. HDlive flow with HDlive silhouette mode in diagnosis of molar pregnancy. Ultrasound Obstet Gynecol 2018;52(4):552-554. DOI: 10.1002/uog.19106.

16. Yamashita T, Tenkumo C, Ishibashi M, et al. HDlive flow for diagnosis of invasive mole. Donald School J Ultrasound Obstet Gynecol 2019;13(1):1-3. DOI: 10.5005/jp-journals-10009-1579.

17. Hata T, Mori N, AboEllail MAM, et al. Advances in color Doppler in obstetrics. J South Asian Feder Obst Gynae 2019;11(1):1-12. DOI: 10.5005/jp-journals-10006-1641.

18. Hata T, Mori N, Yamamoto K, et al. SlowflowHD for detection of small fetal peripheral vasculature. Donald School J Ultrasound Obstet Gynecol 2019;13(4):155-158. DOI: 10.5005/jp-journals-10009-1603.

19. Hata T, Koyanagi A, Yamanishi T, et al. Fetal abdominal blood vessels and organ microvasculature detected by SlowflowHD. Ultrasound Obstet Gynecol 2020. DOI: 10.1002/uog.22043.

20. Mankuta D, Nadjari M, Pomp G. Isolated fetal intra-abdominal umbilical vein varix. Clinical importance and recommendations. J Ultrasound Med 2011;30(2):273-276. DOI: 10.7863/jum.2011.30.2.273.

21. Bas-Lando M, Rabinowitz R, Sanueloff $A$, et al. The prenatal diagnosis of isolated fetal varix of the intra-abdominal umbilical vein is associated with favorable neonatal outcome at term: a case series. Arch Gynecol Obstet 2013;288(1):33-39. DOI: 10.1007/s00404-0132743-x. 